A Bayesian, generalized frailty model for comet assays

Peer-reviewed author version

HABTEAB GHEBRETINSAE, Aklilu; FAES, Christel; MOLENBERGHS, Geert; De Boeck, Marlies \& GEYS, Helena (2013) A Bayesian, generalized frailty model for comet assays. In: AAPS Journal, 11, p. 449-455.

DOI: $10.1080 / 10543406.2012 .756499$

Handle: http://hdl.handle.net/1942/14941 


\title{
A Bayesian, Generalized Frailty Model for Comet Assays
}

\author{
Aklilu Habteab Ghebretinsae $^{1} \quad$ Christel Faes $^{1}$ \\ Geert Molenberghs ${ }^{1,2} \quad$ Marlies De Boeck $^{3}$ \\ Helena Geys ${ }^{3,1}$ \\ 1 I-BioStat, Universiteit Hasselt, B-3590 Diepenbeek, Belgium \\ 2 I-BioStat, Katholieke Universiteit Leuven, B-3000 Leuven, Belgium \\ 3 Janssen Pharmaceutica, Turnhoutseweg 40, B-2430 Beerse, Belgium
}

\begin{abstract}
This paper proposes a flexible modeling approach for so-called comet assay data regularly encountered in pre-clinical research. While such data consist of non-Gaussian outcomes in a multi-level hierarchical structure, traditional analyses typically completely or partly ignore this hierarchical nature by summarizing measurements within a cluster. Non-Gaussian outcomes are often modeled using exponential family models. This is true not only for binary and count data, but also for, e.g., time-to-event outcomes. Two important reasons for extending this family are: (1) the possible occurrence of overdispersion, meaning that the variability in the data may not be adequately described by the models which often exhibit a prescribed mean-variance link, and (2) the accommodation of a hierarchical structure in the data, owing to clustering in the data. The first issue is dealt with through so-called overdispersion models. Clustering is often accommodated through the inclusion of random subject-specific effects. Though not always, one conventionally assumes such random effects to be normally distributed. In the case of time-to-event data, one encounters, for example, the gamma frailty model (Duchateau and Janssen 2007). While both of these issues may occur simultaneously, models combining both are uncommon. Molenberghs et al (2010) proposed a broad class of generalized linear models accommodating overdispersion and clustering through two separate sets of random effects. Here, we use this method to model data from a comet assay with a three-level hierarchical structure. Whereas a conjugate gamma random effect is used for the overdispersion random effect, both gamma and Normal random effects are considered for the hierarchical random effect. Apart from model formulation, we place emphasis on Bayesian estimation. Our proposed method has upper hand over the traditional analysis in that it: (1) uses the appropriate distribution stipulated in the literature; (2) deals with the complete hierarchical nature; and (3) uses all information instead of summary measures. The fit of the model to the comet assay is compared against the background of more conventional model fits. Results indicate the toxicity of 1,2-Dimethylhydrazine dihydrochloride at different dose levels (low, medium, and high).
\end{abstract}

Some Keywords: Frailty; Hierarchical model; Random effect; Weibull model. 


\section{Introduction}

The comet assay is a technique used to assess the genotoxic potential of a compound by means of its ability to induce DNA damage in organ cells of male rats. Because the comet assay is quick, sensitive, and cheap, the assay is now widely used and a number of protocols have been developed for use in different types of investigations (Lovell and Omori 2008). However, the statistical analysis of such a comet assay is complicated because of several issues in the data. In this paper, a method is proposed accounting for different challenges: the multi-level structure of the data, the type of data, and the skewness of the outcome of interest.

In a typical comet assay study, a set of cells from exposed animals are investigated for DNA damage. This is done by considering the migration of DNA fragments out of the nucleus after electropheresism which induces typical comet-like structures. Details on the comet assay are described in Section 2. In many protocols, the cells from a single animal are placed on a number of slides. Each cell is then investigated for DNA damage by measuring the tail length and tail intensity of the comet. Because variability is expected between slides and between animals, as illustrated in Section 3, this needs to be taken into account in the statistical analysis. This results in three-level hierarchies, with clustering at the animal and slide level (Figure 4).

Moreover, exploration of the distribution of the gathered data and previous work in this area indicate that the distribution for the responses (tail length and tail intensity) are asymmetric (Lovell and Omori 2008). This is often completely or partly ignored in traditional analyses. The standard approach of modeling non-normal data, such as the tail intensity and tail length in the comet assay is using a generalized linear model (e.g., a Weibull model). The generalized linear model framework (McCullagh and Nelder 1989) is a very rich one. Nevertheless, already in the univariate case, it is well known that many standard members of the family may exhibit overdispersion. This results from the fact that various commonly used members prescribe a relationship between mean and variance. For example, in the Poisson model for count data, mean and variance are equal. In the exponential and Weibull cases, there is a quadratic relationship between them, etc. This is why many proposals have been made to extend the models such that they can deal with so-called overdispersion, which is taken to mean that the actual relationship between mean and variance is different from the one 
prescribed. Overviews are given by Molenberghs, Verbeke, and Demétrio (2007) for the count-data case and, more generally, by Molenberghs et al (2010).

Here, a model is proposed accounting for both the overdispersion in the data and the hierarchical design of the assay. One way forward is by using random effects. Random effects are broadly used to analyze outcomes collected in a repeated-measures, longitudinal, clustered, or multivariate fashion. Random effects can also be used to accommodate the overdispersion in the data. For example, when parameters in the Weibull model are thought of as being random and each observation is drawn from a different Weibull distribution, this would lead to an overdispersed Weibull model. An overview is given in Molenberghs et al (2010). Random effects are frequently assumed to be normal, but they can take various distributional forms, such as beta random effects with binomial data, gamma random effects with count data, etc. An illustrious counterexample is time-to-event data where gamma random-effects, usually termed gamma frailties, are in common use. Molenberghs et al (2010) proposed an extended framework where both types of random effects are considered simultaneously, so as to deal, at the same time, with overdispersion on the one hand and data hierarchies on the other.

Arguably, such model development, while requiring additional work, is necessary for a number of reasons. First, Molenberghs, Verbeke, and Demétrio (2007) showed that classical generalized linear mixed models (GLMM) can be inadequate to model, at the same time, overdispersion and data hierarchies. Precisely, they modeled repeatedly measured epileptic-seizure data and found that the more conventional GLMM exhibited inferior fit, but also that two types of inferences were incorrect under the simpler model: (1) the correlation between repeated measures was substantially overestimated with the GLMM and (2) the treatment effect with the GLMM was found significant whereas the extended model showed that there was no treatment effect at all. Thus, the spurious treatment effect was entirely a consequence of model misspecification. Second, the design considered here is even more complex, with various hierarchical levels; it is generally inappropriate to consider a model that does not fully accommodate the design. Third, even if the model could be simplified to a more conventional model, this cannot be uncovered without considering a more general model. Thus, the model development proposed here can be used additionally as a goodness-of-fit tool for, say, the 
GLMM.

Here, we will focus on the specific case of a non-negative continuous outcome in view of the comet assay. Whereas Molenberghs et al (2010) considered a two-level hierarchy in the form of repeated measures on the same subject, the comet assay data exhibit higher-order hierarchies.

In essence, the comet assay represents a hierarchical design with animals nested within doses, a number of slides per animals and several cells measured per slide. Comet measures from an animal are clearly not normally distributed but are rather asymmetric, skewed, bi- or multimodal, a mixture of different distributions, etc. The complications that arise from the complex distributions of comet endpoints are avoided in most standard analyses through the use of the central limit theorem. While the original data at the cell level may not be normally distributed, mean (or median) summaries at slide or animal level will be approximately normally distributed (given the typically large sample sizes) and are thus amenable to standard statistical analyses. Hierarchical or multilevel models make use of information on the various levels of variability but may be quite complex in terms of the distribution between cells of the same animals and difficult to interpret and explain. Their advantage, however, is that they provide estimates of the variability at each level and make use of the information at the cell level thus increasing the power of the study especially if the between-animal variability is not too large.

Also, these authors considered maximum likelihood estimation, but here we rather propose a Bayesian approach. Not only does it have computational advantages, it allows to take relevant information from preceding studies into account, a so-called Bayesian learning approach. The interest here is to see the toxicity of 1,2-Dimethylhydrazine dihydrochloride at the different dose levels (low, medium, and high) using the appropriate distribution and taking in to account the complete hierarchical nature.

The paper is organized as follows. In Section 2, the comet assay data are introduced. Data are explored and the traditional analyses presented in Section 3. Section 4 presents the general framework for combined overdispersion and hierarchical random effects, with then Section 5 zooming in on the non-Gaussian continuous case. Estimation methodology is discussed in Section 6. The effect of overdispersion and clustering is illustrated in Section 7. The data are analyzed in Section 8. 


\section{Comet Assay Data}

The comet assay is a single cell microgel electrophoresis method detecting DNA damage in any target tissue or organ of which a single cell suspension can be prepared. Cells are embedded in agarose, membranes are lysed and proteins extracted. Exposure to high alkali $(\mathrm{pH}>13.0)$ allows expression of single strand breaks and subsequent alkaline electrophoresis ensures migration of DNA fragments out of the nucleus. Visualization of this DNA migration (typical comet-like structures) is performed by a fluorescent dye. An image analysis system coupled to a microscope permits quantification of DNA damage at the single cell level.

Here, the data refer to four groups of six male rats that received a daily oral dose of a compound in three dose levels (low, medium, and high) or vehicle control. On the day of necropsy, an extra group of three animals received a single dose of a positive control $(200 \mathrm{mg} / \mathrm{kg}$ ethyl methanesulfonate, EMS, PC). The animals were sacrificed 3 hours after the last dose administration, their liver was removed and processed for the comet assay. For each animal, a cell suspension is prepared. From each cell suspension, three replicate samples were prepared for scoring. Fifty randomly selected, non-overlapping cells per sample were then scored for DNA damage using a semi-automated scoring system. A total of 150 liver cells were thus scored per animal. DNA damage was assessed by the software system by measuring tail migration, $\%$ tail intensity, and tail moment. Tail migration is the distance from the perimeter of the comet head to the last visible point in the tail; \% tail intensity is the percentage of DNA fragments present in the tail; and tail moment is the product of the amount of DNA in the tail and the mean distance of migration in the tail.

\section{Data Exploration and Traditional Models}

Although for the purpose of comparison across studies, statistical analyses are commonly performed on percentage of tail intensity, tail length is also used. Data for tail intensity and tail length are represented in Figure 1. For these data, an non-negligble set of dispersed observations are encountered and it was more pronounced for tail length. This may require attention in modeling with respect to the adequacy of the model to handle the dispersion present in the data. Further exploration is done 

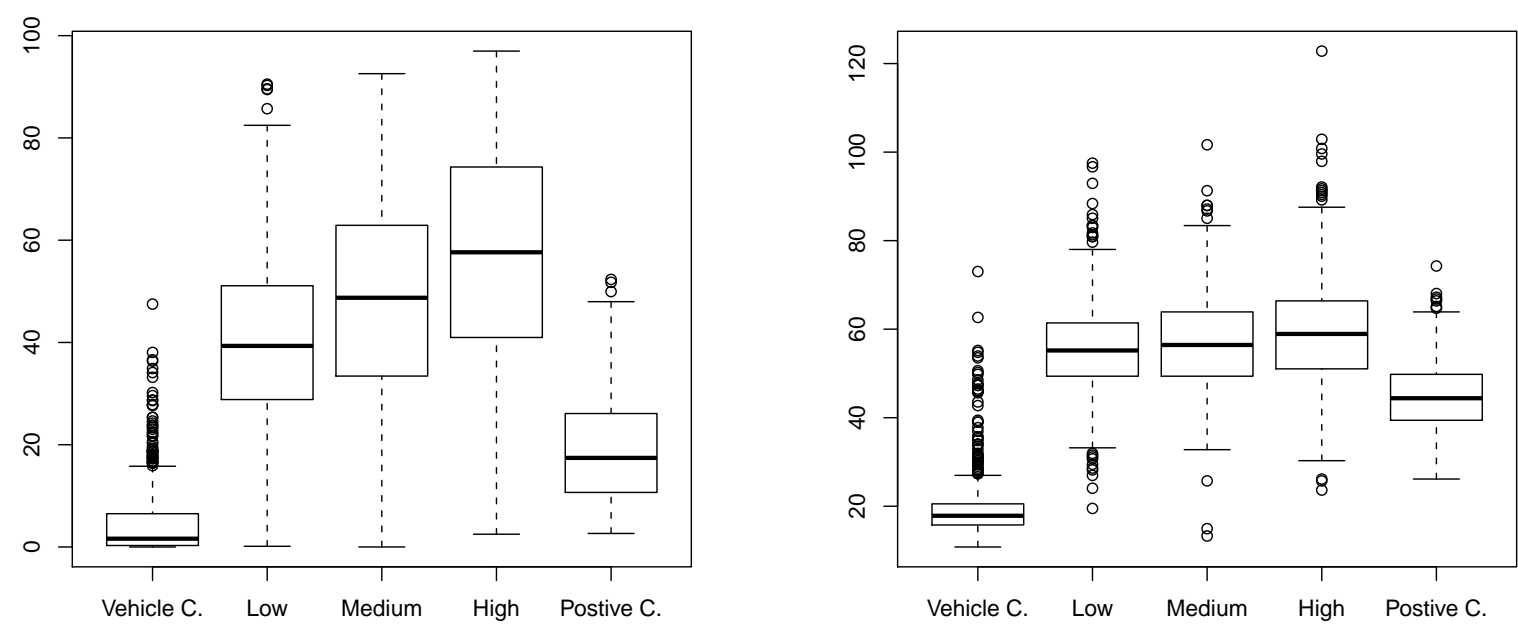

Figure 1: Box plots of tail intensity (left) and tail length (right) at each dose level

to get an idea of the variation at rat- and slide-level. Figure 2 shows scatter plots of the average measurement at rat and slide levels after adjustment of the dose effect. Noticeable variability in the average score of the slides was observed, illustrating the importance of slide effect. Looking at the variability of the averages at slide level before and after adjusting for the rat effect, it can be seen that the variation shrinks more for tail length, implying that the rat effect could be more important for tail length as compared to tail intensity. This suggests the use of more elaborate models to formally check the importance of clustering and overdispersion.

Standard methods to investigate the dose-response relationship of tail length and tail intensity, are based on first log-transforming the outcome to deal with the skewness of the outcome, and second, taking animal-averages of the log-transformed outcomes as a summary measure of the measurements in the animals. Thus, the hierarchical structure is completely ignored and the analysis is done using simple analysis of variance techniques. Sometimes, summary measures for the cells at slide-level are used instead of at the animal-level (Lovell and Omori 2008). The analysis is then performed using a mixed model, fitting the group as a fixed effect and animal as a random effect, and using the Kenward-Roger method for calculating degrees of freedom (Kenward and Roger 1997). However, 

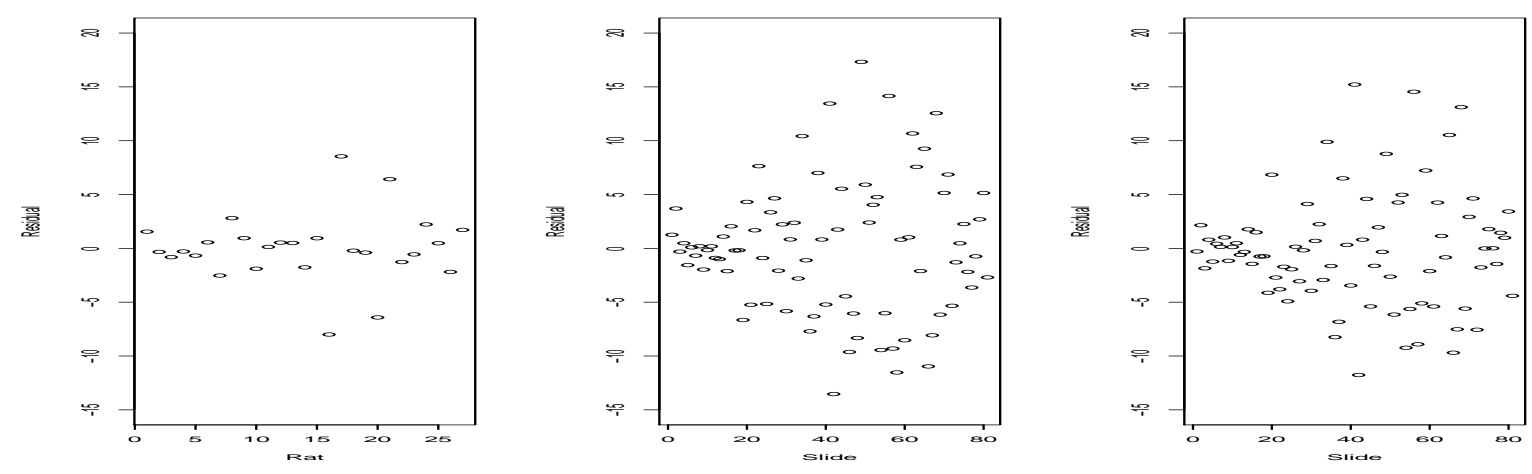

(a) Tail Intensity
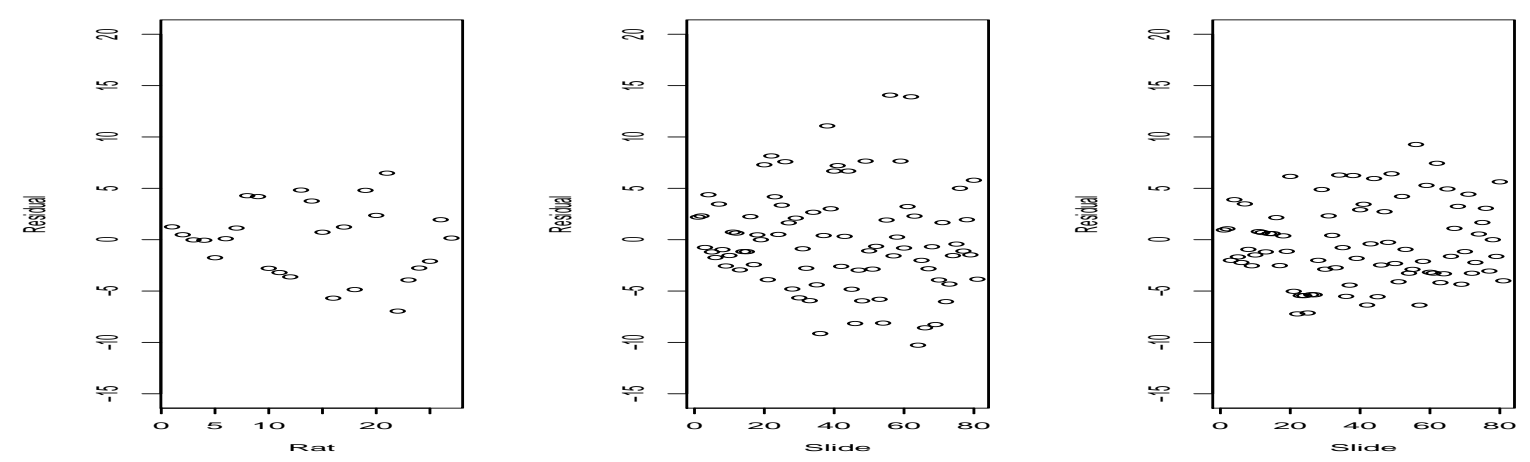

(b) Tail Length

Figure 2: Scatter plot of average tail intensity and tail length after adjusting for the dose effect. (a) Left: at rat level; (b) Middle: at slide level; (c) Right: at slide level, but adjusted for the rat effect.

with this method, one loses a lot of information. Indeed, 150 cell observations are summarized by, for example, a single value. Such averaging effect may have a major impact on parameter estimation and corresponding inferences. Therefore, it is of paramount importance to deal with the full hierarchical structure using an appropriate probability distribution suggested in the literature. The results of the traditionally used analysis of variance and classical Weibull model for tail intensity are presented in Table 1, which will be compared to estimates from the proposed model in Section 8. 
Table 1: Parameter estimates from the conventional models for tail intensity.

\begin{tabular}{lccccc}
\hline \hline & & \multicolumn{2}{c}{ Weibull } & \multicolumn{2}{c}{ Analysis of variance } \\
\hline Effect & Parameter & Estimate(s.e.) & $95 \%$ C.I. & Estimate(s.e.) & $95 \%$ C.I. \\
\hline Vehicle & $\beta_{0}$ & $-2.431(0.056)$ & {$[-2.54,-2.32]$} & $0.234(0.052)$ & {$[0.13,0.34]$} \\
Low versus vehicle & $\beta_{1}$ & $-2.698(0.053)$ & {$[-2.80,-2.59]$} & $3.351(0.073)$ & {$[3.21,3.49]$} \\
Medium versus vehicle & $\beta_{2}$ & $-2.947(0.054)$ & {$[-3.05,-2.84]$} & $3.527(0.074)$ & {$[3.38,3.67]$} \\
High versus vehicle & $\beta_{3}$ & $-3.156(0.055)$ & {$[-3.27,-3.05]$} & $3.693(0.074)$ & {$[3.55,3.84]$} \\
Pos. control versus vehicle & $\beta_{4}$ & $-1.711(0.060)$ & {$[-1.83,-1.59]$} & $2.543(0.09)$ & {$[2.37,2.73]$} \\
Weibull shape & $\rho$ & $1.376(0.018)$ & {$[1.34,1.41]$} & & \\
\hline \hline
\end{tabular}

\section{Models Combining Conjugate and Normal Random Effects}

The following general model family was proposed by Molenberghs et al (2010) for modeling overdispersed and correlated data:

$$
f_{i}\left(y_{i j} \mid \boldsymbol{b}_{\boldsymbol{i}}, \boldsymbol{\xi}\right)=\exp \left\{\phi^{-1}\left[y_{i j} \lambda_{i j}-\psi\left(\lambda_{i j}\right)\right]+c\left(y_{i j}, \phi\right)\right\}
$$

for outcome $y_{i j}$ on subject $i=1, \ldots, N$ at occasion $j=1, \ldots, n_{i}$. The unknown parameters $\lambda_{i j}$ and $\phi$ are often termed natural parameter and scale parameter, respectively. The term $c\left(y_{i j}, \phi\right)$ is the normalizing constant. The function $\psi(\cdot)$ is a known function with the property that $E\left[y_{i j} \mid \boldsymbol{b}_{\boldsymbol{i}}, \boldsymbol{\xi}\right]=$ $\psi^{\prime}\left(\lambda_{i j}\right)$ and $\operatorname{var}\left(y_{i j} \mid \boldsymbol{b}_{\boldsymbol{i}}, \boldsymbol{\xi}\right)=\phi \psi^{\prime \prime}\left(\lambda_{i j}\right)$. Model specification proceeds by assuming that the conditional mean of $y_{i j}$ is given by

$$
E\left(Y_{i j} \mid \theta_{i j}, \boldsymbol{b}_{\boldsymbol{i}}\right)=\mu_{i j}^{c}=\theta_{i j} \kappa_{i j}
$$

where $\theta_{i j} \sim \mathcal{G}_{i j}\left(\xi_{i j}, \sigma_{i j}^{2}\right)$ for some distribution $\mathcal{G}_{i j}$ with mean $\xi_{i j}$ and variance $\sigma_{i j}^{2}$ and $\kappa_{i j}=g\left(\eta_{i j}\right)=$ $g\left(\boldsymbol{x}_{i j}^{\prime} \boldsymbol{\xi}+\boldsymbol{z}_{i j}^{\prime} \boldsymbol{b}_{\boldsymbol{i}}\right)$ for some function $g$ and $\boldsymbol{b}_{\boldsymbol{i}} \sim N(\mathbf{0}, D)$. The random variable $\theta_{i j}$ is used to account for the overdispersion in the data, while the random effect in $\kappa_{i j}$ accounts for the clustered or hierarchical structure in the data. The two parameters $\eta_{i j}$ and $\lambda_{i j}$ refer to the linear predictor and/or the natural parameter. The basic difference is $\lambda_{i j}$ encompasses the random variables $\theta_{i j}$, whereas $\eta_{i j}$ refers to the 'GLMM part' only. 
It is convenient, but not strictly necessary, to assume that the two sets of random effects, $\boldsymbol{\theta}_{i}$ and $\boldsymbol{b}_{\boldsymbol{i}}$, are independent of each other. Regarding the components $\theta_{i j}$ of $\boldsymbol{\theta}_{i}$, three useful special cases result from assuming that: (1) they are independent; (2) they are correlated, implying that the collection of univariate distributions $\mathcal{G}_{i j}\left(\xi_{i j}, \sigma_{i j}^{2}\right)$ needs to be replaced with a multivariate one; and (3) they are equal to each other, useful in applications with exchangeable outcomes $Y_{i j}$.

Parameterization (2) allows for random effects $\theta_{i j}$ capturing overdispersion, and formulated directly at mean scale, whereas $\kappa_{i j}$ could be considered the generalized linear mixed model component (Molenberghs and Verbeke 2005). The relationship between mean and natural parameter now is

$$
\lambda_{i j}=h\left(\mu_{i j}^{c}\right)=h\left(\theta_{i j} \kappa_{i j}\right) .
$$

Standard GLM ideas can be applied to derive the mean and variance, combined with iteratedexpectation-based calculations. For the mean, it follows that

$$
E\left(Y_{i j}\right)=E\left(\theta_{i j}\right) E\left(\kappa_{i j}\right)=E\left[h^{-1}\left(\lambda_{i j}\right)\right] .
$$

For the Weibull-Exponential case, (4) allows for explicit expressions of moments and marginal densities.

An important concept is conjugacy, in the sense of Cox and Hinkley (1974, p. 370) and Lee, Nelder, and Pawitan (2006, p. 178). Conjugacy refers to the fact that the hierarchical and random-effects densities have similar algebraic forms. Conjugate distributions produce a general and closed-form solution for the corresponding marginal distribution. Molenberghs et al (2010) adapted conjugacy to the situation where both normal and overdispersion random effects are included. This property aims at maintaining conjugacy across the presence of normally distributed random effects. It has been shown that the Weibull model, upon transformation, and in particular the exponential model, not only enjoys conjugacy but, in its extended form, also strong conjugacy. For detailed explanation on the combined model, concept of conjugacy, expression of moments and marginal densities, we refer to Molenberghs et al (2010). 


\section{Hierarchical, Overdispersed, Non-Gaussian Continuous Outcomes}

In this section, the model of interest for the comet assay data will be outlined. Because tail intensity and tail length are skewed, non-negative and continuous, which is similar to time-to-event data, an exponential or Weibull model appears appropriate. It is well known that the exponential model with gamma random effects is conjugate. The same holds for the Weibull model, when considered exponential in the outcome $y_{i j}^{\rho}$. These facts are reviewed in Molenberghs et al (2010). In particular, closed form expressions can be derived for the joint distribution, mean, variance, and higher-order moments.

Let us first assume that there is only one level of hierarchy in the data, e.g., the variability between animals. We then propose to use a combined model with a normal random effect to handle the hierarchy in the data and a conjugate random effect to account for overdisperion in the response. Using the Weibull distribution, this leads to the following specification of the model:

$$
\begin{aligned}
y_{i j} \mid b_{i}, \theta_{i j} & \sim \operatorname{Weibull}(\rho, \kappa), \\
\kappa & =\lambda \theta_{i j} \exp \left(\boldsymbol{x}_{i j}^{\prime} \boldsymbol{\xi}+\boldsymbol{z}_{i j}^{\prime} \boldsymbol{b}_{\boldsymbol{i}}\right), \\
b_{i} & \sim \operatorname{Normal}(0, D), \\
\theta_{i j} & \sim \operatorname{Gamma}(\alpha, \beta),
\end{aligned}
$$

with $b_{i}$ the animal-specific random effects to account for the clustering of observations and $\theta_{i j}$ the measurement-specific random effects to accommodate for overdispersion. This model can be expressed as

$$
\begin{aligned}
f\left(\boldsymbol{y}_{i} \mid \boldsymbol{\theta}_{i}, \boldsymbol{b}_{\boldsymbol{i}}\right) & =\prod_{j=1}^{n_{i}} \lambda \rho \theta_{i j} y_{i j}^{\rho-1} e^{\eta_{i j}} e^{-\lambda y_{i j}^{\rho} \theta_{i j} e^{\eta_{i j}}}, \\
\eta_{i j} & =\boldsymbol{x}_{i j}^{\prime} \boldsymbol{\xi}+\boldsymbol{z}_{i j}^{\prime} \boldsymbol{b}_{\boldsymbol{i}} \\
f\left(\boldsymbol{\theta}_{i}\right) & =\prod_{j=1}^{n_{i}} \frac{1}{\beta_{j}^{\alpha_{j}} \Gamma\left(\alpha_{j}\right)} \theta_{i j}^{\alpha_{j}-1} e^{-\theta_{i j} / \beta_{j}}, \\
f\left(\boldsymbol{b}_{\boldsymbol{i}}\right) & =\frac{1}{(2 \pi)^{q / 2}|D|^{1 / 2}} e^{-\frac{1}{2} \boldsymbol{b}_{\boldsymbol{i}}{ }^{\prime} D^{-1} \boldsymbol{b}_{\boldsymbol{i}}} .
\end{aligned}
$$

A few comments are in place. First, it is implicit that the gamma random effects are independent. 
This need not be the case and, like in the Poisson case, extension via multivariate gamma distributions is possible. Second, setting $\rho=1$ leads to the special case of an exponential time-to-event distribution. Third, it is evident that the classical gamma frailty model (i.e., no normal random effects) and the Weibull-based GLMM (i.e., no gamma random effects) follow as special cases. Fourth, owing to the conjugacy result mentioned above and the following property of the gamma distribution:

$$
\frac{1}{\kappa} f(\theta \mid \alpha, \beta)=\frac{1}{\kappa} \frac{1}{\beta^{\alpha} \Gamma(\alpha)} \theta^{\alpha-1} e^{-\theta / \beta}=\frac{1}{(\kappa \beta)^{\alpha} \Gamma(\alpha)}(\kappa \theta)^{\alpha-1} e^{-(\kappa \theta) /(\kappa \beta)}=f(\kappa \beta \mid \alpha, \kappa \beta),
$$

strong conjugacy applies. This is typically considered for the exponential model, but it holds for the Weibull model too, merely by observing, as stated above, that the Weibull model is nothing but an exponential model for the random variable $Y_{i j}^{\rho}$. It is equally possible to derive this result by merely re-writing the factor $\phi=\lambda \kappa$. Fifth, the above expressions are derived for a two-parameter gamma density. It is customary in a gamma frailty context (Duchateau and Janssen 2007) to set $\alpha_{j} \beta_{j}=1$, for reasons of identifiability. In this case, (7) is replaced by

$$
f\left(\boldsymbol{\theta}_{i}\right)=\prod_{j=1}^{n_{i}} \frac{1}{\left(\frac{1}{\alpha_{j}}\right)^{\alpha_{j}} \Gamma\left(\alpha_{j}\right)} \theta_{i j}^{\alpha_{j}-1} e^{-\alpha_{j} \theta_{i j}},
$$

Alternatively, assuming $\alpha_{j}=1$ and $\beta_{j}=1 / \delta_{j}$, one could write

$$
f\left(\boldsymbol{\theta}_{i}\right)=\prod_{j=1}^{n_{i}} \delta_{j} e^{-\delta_{j} \theta_{i j}},
$$

implying that the gamma density is reduced to an exponential one.

Next, let us propose an extension of the above model accounting for an extra level of hierarchy. Indeed, in the comet assay there are two sources of variation: one coming from the slide effect and one from the animal effect. The previous model can be extended by the use of three random effects of which one is the overdispersion effect. In addition, while typically a normal random effect is included in the linear predictor to account for the clustering, as in Molenberghs et al (2010), also a multiplicative factor using a multivariate gamma distribution can be used, similar to the multiplicative factor for the overdispersion random effect. For example, let us consider a model with a normally distributed random effect for the first hierarchy in the data and a gamma random effect for the second hierarchy in the data. In addition, we allow for the overdispersion in the model via another gamma-random effect. Let the outcome $Y_{i j k}$ be the measurement for unit $k=1, \ldots, n_{i j}$ of cluster 
$i=1, \ldots, N$, sub-cluster $j=1, \ldots, n_{i}$. The model can then be expressed as:

$$
\begin{aligned}
Y_{i j k} \mid b_{i}, b_{i j}, \theta_{i j k} & \sim \operatorname{Weibull}(\rho, k), \\
k & =\lambda \theta_{i j k} b_{i j} \exp \left(\boldsymbol{x}_{i j}^{\prime} \boldsymbol{\xi}+\boldsymbol{z}_{i j}^{\prime} \boldsymbol{b}_{\boldsymbol{i}}\right), \\
\theta_{i j k} & \sim \operatorname{Gamma}\left(\alpha_{1}, 1 / \alpha_{1}\right), \\
b_{i j} & \sim \operatorname{Gamma}\left(\alpha_{2}, 1 / \alpha_{2}\right), \\
b_{i} & \sim \operatorname{Normal}(0, D),
\end{aligned}
$$

leading to

$$
\begin{aligned}
f\left(y_{i j k} \mid \theta_{i j k}, b_{i}, b_{i j}\right) & =\lambda \rho \theta_{i j k} b_{i j} y_{i j k}^{\rho-1} e^{\boldsymbol{x}_{i j k}^{\prime} \boldsymbol{\xi}+b_{i}} e^{-\lambda y_{i j k}^{\rho} \theta_{i j k} b_{i j} e^{\boldsymbol{x}_{i j k}^{\prime} \boldsymbol{\xi}_{+b_{i}}},} \\
f\left(\theta_{i j k}\right) & =\frac{1}{\left(\frac{1}{\alpha_{1}}\right)^{\alpha_{1}} \Gamma\left(\alpha_{1}\right)} \theta_{i j k}^{\alpha_{1}-1} e^{-\alpha_{1} \theta_{i j k}}, \\
f\left(b_{i}\right) & =\frac{1}{(2 \pi d)^{1 / 2}} e^{-\frac{1}{2 d} b_{i}^{2}}, \\
f\left(b_{i j}\right) & =\frac{1}{\left(\frac{1}{\alpha_{2}}\right)^{\alpha_{2}} \Gamma\left(\alpha_{2}\right)} b_{i j}^{\alpha_{2}-1} e^{-\alpha_{2} b_{i j}},
\end{aligned}
$$

The conditional mean, given the overdispersion and hierarchical random effects is:

$$
E\left(y_{i j k} \mid \theta_{i j k}, b_{i}, b_{i j}\right)=\frac{\Gamma\left(\frac{1}{\rho}+1\right)}{\lambda \theta_{i j k} b_{i j} e^{\boldsymbol{x}_{i j k}^{\prime} \boldsymbol{\xi}+b_{i}}},
$$

Similarly, other models can be defined where either a gamma or a normal random effect is considered. This results in four different models: (a) Weibull Gamma(OD) Normal(RE1) Normal(RE2); (b) Weibull Gamma(OD) Normal(RE1) Gamma(RE2); (c) Weibull Gamma(OD) Gamma(RE1) Normal(RE2); and (d) Weibull Gamma(OD) Gamma(RE1) Gamma(RE2), where (.) explains what this random effect is considered for: OD refers to the overdispersion random effect, RE1 and RE2 refer to the first and second hierarchical random effect, respectively. As a result, a very flexible modeling framework is obtained for which model selection can easily be performed.

\section{Bayesian Estimation Using MCMC}

In the Bayesian framework, computation of the posterior probability is of main interest. The posterior probabilities are obtained by updating the likelihood with prior probabilities. For the Weibull- 
Gamma(OD)-Normal(RE1)-Gamma(RE2) model for instance, combining the distribution of the outcome variable given in (12) with prior densities $f\left(\vartheta, \theta_{i j k}, b_{i}, b_{i j}\right)$, the posterior density is:

$$
p(\boldsymbol{\vartheta} \mid y, x) \propto \prod_{i=1}^{N} \prod_{j=1}^{J} \prod_{k=1}^{n} f\left(y_{i j k} \mid \lambda_{i j k}\right) f\left(\boldsymbol{\vartheta}, \theta_{i j k}, b_{i}, b_{i j}\right),
$$

where $\lambda_{i j k}=\theta_{i j k} b_{i j} \exp \left(\boldsymbol{x}_{i j k}^{\prime} \boldsymbol{\xi}+b_{i}\right)$ and $\boldsymbol{\vartheta}$ is a group of parameters $(\boldsymbol{\xi}, \rho)$.

Inference is typically made by taking random draws from this posterior density using Markov chain Monte Carlo simulation (MCMC), particularly the Gibbs sampling. The basic idea of Gibbs sampling is to partition the set of unknown parameters and then estimate them one at a time or in a group, conditional on all others. The Gibbs sampler starts with initial values for all parameters and then updates them in turn, giving each a random estimate based on the data and the current guess of the other parameters in the model (Gelman and Hill 2006). Sampling was done in two chains and dispersed initial values were given for all parameters in the two chains. 150,000 samples were drawn from each chain and the first 100,000 samples were discarded. To ensure the samples are drawn from the target posterior density, convergence was checked by comparing the between- and within-chain variation for each parameter in the simulated samples.

For this analysis, non-informative or weak priors were used for all the parameters of interest: $\beta_{0}, \beta_{1}, \beta_{2}, \beta_{3}, \beta_{4} \sim N\left(0,10^{6}\right) ; \sigma_{r}^{2} \sim I G(0.1,0.001) ; \rho \sim \exp (0.01) ; \alpha_{\theta} \sim \operatorname{Gamma}(2,2) ;$ and $\alpha_{s} \sim \operatorname{Gamma}(0.1,0.1)$, where IG is the Inverse Gamma distribution. The mean was reported as point estimate for each parameter, together with the 95 percent credible interval that ranges between the 2.5 and 97.5 percent quantiles. Note that, while $\alpha=0.1$ for the gamma distribution will result in a relatively informative prior, given the size of the dataset, varying this value has little or no impact on the conclusion. For relatively small sets of data, however, caution is needed and it would be wise to undertake a sensitivity analysis towards the prior assumptions made.

\section{Illustration of Overdispersion and of the Clustering Effect}

Overdispersed data, in which the variability in the data is beyond the variance of the model considered, occurs quite often in practice. This is basically because of the restricted relationship between mean and variance functions. The extra-variability could be due to some unaccounted covariates/factors, 

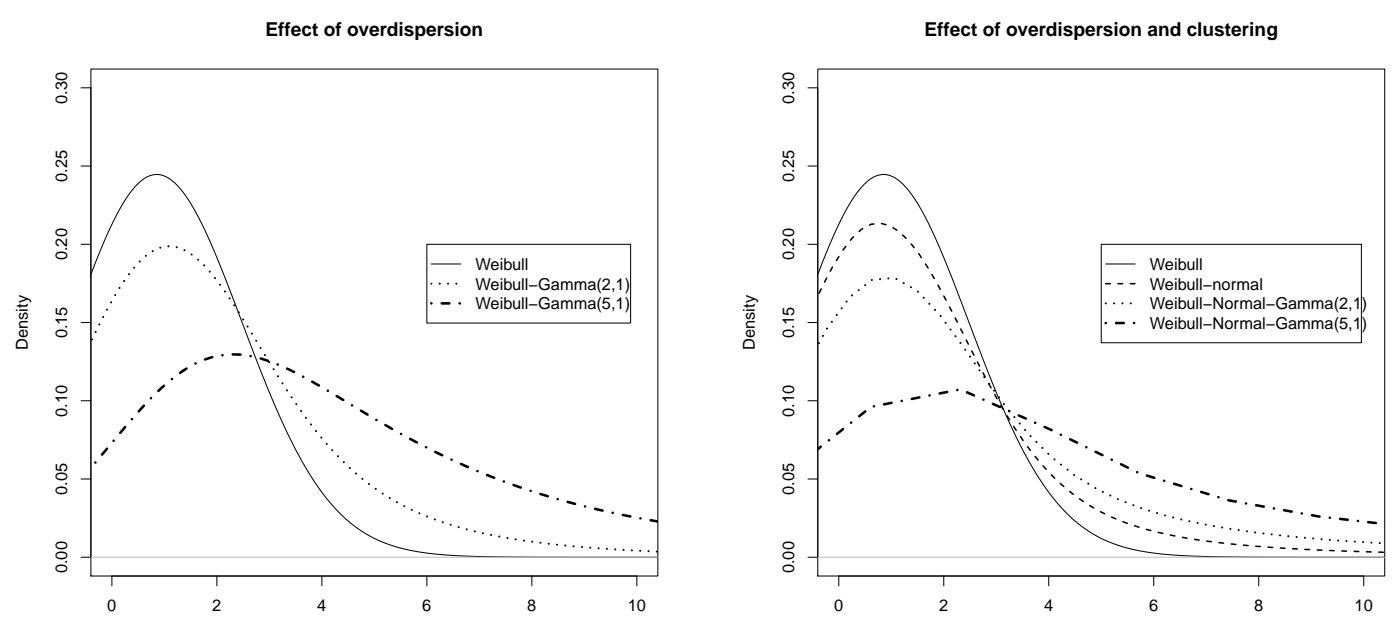

Figure 3: Illustration of the effect of clustering and overdispersion.

heterogeneous population, clustering effect and many others. The extra unaccounted variability can be accounted for by the use of mixture models or using an overdispersion parameter. In this case, a continuous overdispersion random effect is used. We now illustrate how the effect of the overdispersion random effect and the clustering random effect extend the Weibull model to accommodate more dispersed data. This is illustrated by simulating data from models that accounts both the overdispersion and the clustering random effect, model that considers either of the two random effects and models that considers neither of them. Let us first see the effect of overdispersion alone. The set of data are generated from: (1) Weibull Model: Weibull $(\rho, 1)$ and (2) Weibull-Gamma Model: Weibull $(\rho, \theta)$. In the Weibull-Gamma model, we assume different choices for the $\operatorname{Gamma}(\alpha, 1)$ distribution (different choices of $\alpha$ parameters) in order to see the effect of overdispersion. Similarly, to have an idea of the effect of clustering alone and the effect overdispersion combined with clustering, we generate data from the following set: (1) Weibull Model: Weibull $(\rho, 1)$; (2) Weibull-Normal Model: Weibull $\left(\rho, e^{b_{i}}\right)$; and (3) Weibull-Gamma-Normal Model: Weibull $\left(\rho, \theta e^{b_{i}}\right)$, where $\rho=1.4$, $b_{i} \sim \operatorname{Normal}(0,1), i=1, \ldots, 20$, and $\theta \sim \operatorname{Gamma}(\alpha, 1)$.

The density plot of the data generated (Figure 3) indicates that with the inclusion of an overdispersion random effect, the data were overdispersed. Also, introducing clustering led to more dispersion. With the inclusion of both the overdispersion and clustering random effect it was even dispersed more and 


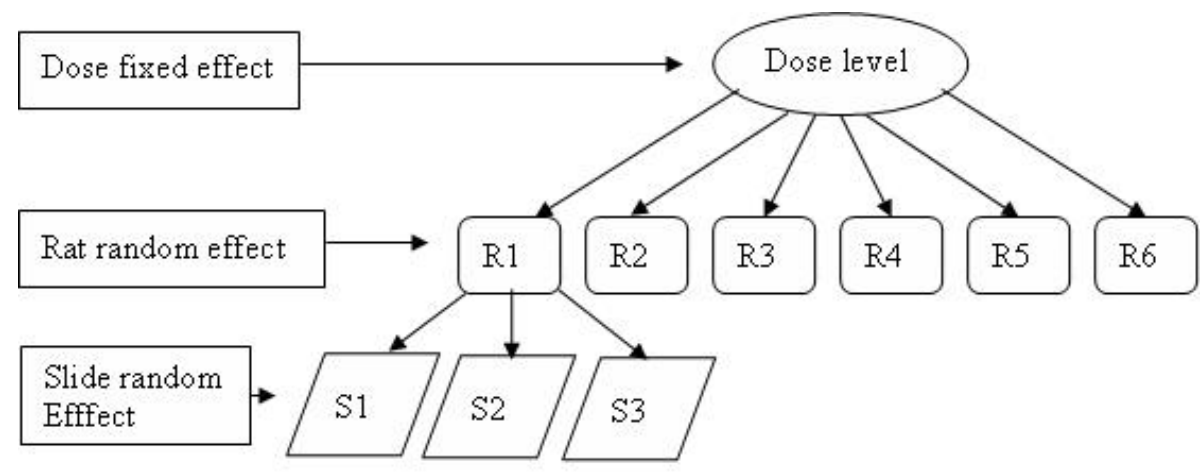

Figure 4: Hierarchical structure of the design.

the degree of dispersion depends on the $\alpha$ parameter. If this is the case, analyzing more dispersed data using the traditional model which do not address overdispersion and clustering may leave a lot of variability unexplained and it can affect the precision of the estimates.

\section{Application to the Comet Assay Data}

The primary goal is to assess the toxicity of 1,2-Dimethylhydrazine dihydrochloride at different dose levels. The data described in Section 2 are analyzed taking the multilevel hierarchical nature as illustrated in Figure 4 into account. Cells coming from the same rat could be more alike due to biological reasons, implying clustering at the animal level. Moreover the fact that cells are grouped into three slides could pose some sort of clustering due to uncontrolled differences in external factors such as the amount of gel being used.

In addition to the hierarchical structure, the skewed nature of the outcome variable adds complexity. Tail intensity and tail length are non-negative continuous outcomes. In the literature, a number of probability distributions were proposed for modelling the distribution. These include the Weibull, exponential, logistic, normal, log-normal, and log-logistic distributions (Lovell and Omori 2008). Ejchart and Sadlej-Sosnowska (2003) found that Weibull was the best fit for such data. In our analyses, also the Weibull distribution was assumed. As explained in the previous section, a meanvariance relationship exists in the Weibull case, unlike in the Gaussian case. Hence, variability in the data may not be adequately accounted for by the model, and therefore an overdispersion random 
Table 2: Overview of models considered with DIC for Tail Intensity(TI)and Tail Length(TL)

\begin{tabular}{|c|c|c|c|c|c|c|c|c|}
\hline \multirow[b]{3}{*}{ Model } & \multicolumn{6}{|c|}{ Distribution for } & \multirow[b]{3}{*}{$\operatorname{DIC}(\mathrm{TI})$} & \multirow[b]{3}{*}{$\mathrm{DIC}(\mathrm{TL})$} \\
\hline & \multirow{2}{*}{$\begin{array}{r}\text { Response } \\
\text { Weibull }\end{array}$} & \multirow{2}{*}{$\begin{array}{c}\text { Overdispersion } \\
\text { Gamma }\end{array}$} & \multicolumn{2}{|c|}{ RE1(rat) } & \multicolumn{2}{|c|}{ RE2(slide) } & & \\
\hline & & & Normal & Gamma & Normal & Gamma & & \\
\hline 1 & $\sqrt{ }$ & & & & & & 33869.6 & 30878.8 \\
\hline 2 & $\sqrt{ }$ & & $\sqrt{ }$ & & & & 33823.9 & 30421.6 \\
\hline 3 & $\sqrt{ }$ & & & $\sqrt{ }$ & & & 33823.5 & 30420.2 \\
\hline 4 & $\sqrt{ }$ & $\sqrt{ }$ & & & & & 33895.6 & 27378.5 \\
\hline 5 & $\sqrt{ }$ & $\sqrt{ }$ & $\sqrt{ }$ & & & & 33853.7 & 26901.6 \\
\hline 6 & $\sqrt{ }$ & $\sqrt{ }$ & & $\sqrt{ }$ & & & 33852.5 & 26883 \\
\hline 7 & $\sqrt{ }$ & & & & $\sqrt{ }$ & & 33728.9 & 29622.6 \\
\hline 8 & $\sqrt{ }$ & & & & & $\sqrt{ }$ & 33728.5 & 29620.8 \\
\hline 9 & $\sqrt{ }$ & $\sqrt{ }$ & & & $\sqrt{ }$ & & 33760.7 & 26386.9 \\
\hline 10 & $\sqrt{ }$ & $\sqrt{ }$ & & & & $\sqrt{ }$ & 33760.6 & 26377 \\
\hline 11 & $\sqrt{ }$ & & $\sqrt{ }$ & & $\sqrt{ }$ & & 33728.7 & 29623.4 \\
\hline 12 & $\sqrt{ }$ & & $\sqrt{ }$ & & & $\sqrt{ }$ & 33728.6 & 29619.5 \\
\hline 13 & $\sqrt{ }$ & & & $\sqrt{ }$ & $\sqrt{ }$ & & 33730.3 & 29631.1 \\
\hline 14 & $\sqrt{ }$ & & & $\sqrt{ }$ & & $\sqrt{ }$ & 33729.7 & 29605.2 \\
\hline 15 & $\sqrt{ }$ & $\sqrt{ }$ & $\sqrt{ }$ & & $\sqrt{ }$ & & 33761.6 & 26374.4 \\
\hline 16 & $\sqrt{ }$ & $\sqrt{ }$ & $\sqrt{ }$ & & & $\sqrt{ }$ & 33760.5 & 26333.1 \\
\hline 17 & $\sqrt{ }$ & $\sqrt{ }$ & & $\sqrt{ }$ & $\sqrt{ }$ & & 33760.6 & 26338 \\
\hline 18 & $\sqrt{ }$ & $\sqrt{ }$ & & $\sqrt{ }$ & & $\sqrt{ }$ & 33758.6 & 26209.6 \\
\hline
\end{tabular}

effect parameter is added. A gamma random effect is used for the overdispersion and both normal and gamma random effects are used to explain the hierarchical structure. As described in Section 4, the normal random effect is included in the linear predictor and the gamma random effect is included as multiplicative effect together with the overdispersion factor. This creates a wide choice of models to choose from. Table 2 presents an overview of the models considered.

Model 1 is the traditional Weibull model that ignores the hierarchical nature as well as overdispersion. Model 4 considers the overdispersion but not the hierarchical nature. Models 2, 3, 7, and 8 consider one random effect (rat or slide) and ignore the over-dispersion and the other random effect, the classical gamma frailty model being part of it. Models 5, 6, 9, and 10 consider the overdispersion 
and one random effect but ignore the second one. Models 11-14 consider the correct hierarchical structure but ignore the overdispersion. The last four models account for both the hierarchical nature and overdispersion. Note that the DIC is subject to random variability and hence differences in value by $2-4$ should not be regarded as evidence for a difference. Therefore, Models 7 and 8 on the one hand and Models 11-14 on the other should be regarded as roughly equivalent.

Let $y_{i j k}$ represent the tail intensity or tail length measured for a $k^{t h}$ cell $(k=1, \ldots, 50)$ from rat $i=1, \ldots, 27$ in slide $j=1,2,3$. If we consider the Weibull-Gamma(OD)-Normal(RE1)-Normal(RE2) model, for instance, the $\lambda_{i j k}$ will be:

$$
\lambda_{i j k}=\theta_{i j k} \exp \left(\beta_{0}+\beta_{1} L_{i j k}+\beta_{2} M_{i j k}+\beta_{3} H_{i j k}+\beta_{4} P C_{i}+r_{i}+s_{i j}\right)
$$

with $\theta_{i j k} \sim \operatorname{Gamma}\left(\alpha_{1}, \frac{1}{\alpha_{1}}\right), r_{i} \sim N\left(0, d_{1}\right)$ and $s_{i j} \sim N\left(0, d_{2}\right)$. Here, $L_{i j k}$ is the indicator variable whether rat $i$ is given a Low dose ( 1 if it is given low dose; 0 otherwise). Similarly, $M_{i j k}, H_{i j k}, P C_{i j k}$ are the indicator variables for medium dose, high dose, or positive control, respectively. The random intercept $r_{i}$ corresponds to the rat-specific effect whereas $s_{i j}$ corresponds to the slide-specific effect $j$ of rat $i . \theta_{i j k}$ is the overdispersion random effect. Similarly, the Weibull-Gamma(OD)-Normal(RE1)Gamma(RE2) is parameterized as:

$$
\lambda_{i j k}=\theta_{i j k} * s_{i j} * \exp \left(\beta_{0}+\beta_{1} * L_{i j k}+\beta_{2} * M_{i j k}+\beta_{3} * H_{i j k}+\beta_{4} * P C_{i j k}+r_{i}\right),
$$

with $\theta_{i j k} \sim \operatorname{Gamma}\left(\alpha_{1}, \frac{1}{\alpha_{1}}\right), r_{i} \sim N(0, d)$ and $s_{i j} \sim \operatorname{Gamma}\left(\alpha_{2}, \frac{1}{\alpha_{2}}\right)$. The fixed effect $\beta_{0}$ denotes the control (vehicle) effect. The parameters $\beta_{1}$ to $\beta_{4}$ are the contrasts of interest that represent the effect of low dose, medium dose, high dose, and positive control versus vehicle. All other models follow similarly. The R2winbugs code for Models 8 and 16 is given in Appendix A.

The next issue is model comparison. In situations where non-informative priors are used or when where huge amounts of data are available, the data overwhelm the choice of the prior and Bayesian estimates are equivalent with maximum likelihood estimates. In such a case, the likelihood ratio can be used to formally test hypotheses and compare nested models. In this case, the deviance information criterion (DIC) as a penalty for the complexity of the model is used when comparing models. Based on the deviance, which favors complex models, Weibull-Gamma(OD)-Gamma(RE1)Gamma(RE2) was to be preferred. For tail intensity based on the DIC, Model 8, was the preferred 
Table 3: Parameter estimates obtained from Models 8 [Weibull-Gamma(RE2)] and 12 [WeibullNormal(RE1)-Gamma(RE2)] for tail intensity.

\begin{tabular}{lccccc}
\hline \hline & \multicolumn{4}{c}{ Weibull-Gamma(RE2) } & \multicolumn{2}{c}{ Weib.-Norm.(RE1)-Gamma(RE2) } \\
\hline Effect & Parameter & Est.(s.e.) & $95 \%$ C.I. & Est.(s.e.) & $95 \%$ C.I. \\
\hline Vehicle & $\beta_{0}$ & $-2.419(0.079)$ & {$[-2.57,-2.26]$} & $-2.427(0.085)$ & {$[-2.59,-2.25]$} \\
Low versus vehicle & $\beta_{1}$ & $-2.854(0.097)$ & {$[-3.04,-2.66]$} & $-2.850(0.104)$ & {$[-3.06,-2.65]$} \\
Medium versus vehicle & $\beta_{2}$ & $-3.092(0.098)$ & {$[-3.29,-2.90]$} & $-3.088(0.106)$ & {$[-3.30,-2.88]$} \\
High versus vehicle & $\beta_{3}$ & $-3.317(0.098)$ & {$[-3.51,-3.12]$} & $-3.312(0.107)$ & {$[-3.53,-3.11]$} \\
Pos. control versus vehicle & $\beta_{4}$ & $-1.829(0.115)$ & {$[-2.05,-1.60]$} & $-1.826(0.124)$ & {$[-2.07,-1.58]$} \\
Weibull shape & $\rho$ & $1.420(0.019)$ & {$[1.38,1.46]$} & $1.419(0.019)$ & {$[1.38,1.46]$} \\
Precision of RE1 & $\frac{1}{d}$ & & & $114.2(79.29)$ & {$[28.60,331.61]$} \\
RE2 parameter & $\alpha_{2}$ & $18.33(4.036)$ & {$[11.68,27.3]$} & $19.99(4.493)$ & {$[12.08,29.54]$} \\
\hline \hline
\end{tabular}

model followed by Model 12. Note that the penalty term, measuring the complexity of the model, for the models with overdispersion was large. Looking at the best two models, outperforming Model 8 was not unexpected from the exploratory data analysis for tail intensity, the variability at the slide level did not reduce much after removing the rat effect.

The parameter estimates and 95 percent credible intervals for Models 8 and 12 are presented in Table 3. Models 12 and 8 are nested models, where Model 12 is an extension of model 8 by inclusion of the normal random effect for the animal level. The parameter estimates from both models are very similar and we notice that the standard errors from Model 12 are consistently and slightly higher as opposed to the ones obtained with Model 8. This is in line with the expectation that, with exclusion of one hierarchical level, the effective degrees of freedom is usually overestimated which results in underestimation of the standard errors.

The parameter estimates from the model with overdispersion only (Model 4) had higher standard errors compared to the estimates from a classical Weibull model. In addition, they were lower compared to that of Weibull Gamma(RE2), the preferred model. The estimates from models with both overdispersion and clustering have higher standard errors compared with models with either overdispersion or clustering. 

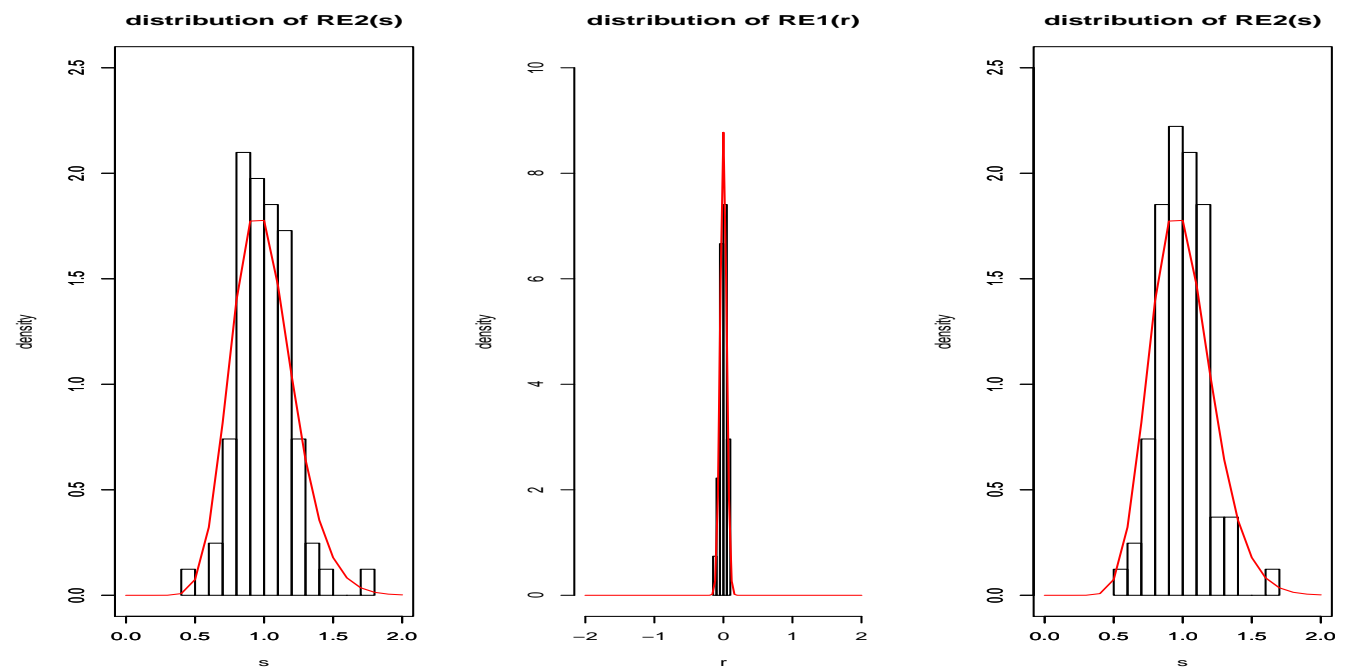

Figure 5: The distribution of the estimated random effects superimposed on the posterior density for Models 8 (Left) and 12 (Middle and Right) for tail intensity.

The $95 \%$ credible interval for $\rho$ did not include 1, which conveys that the Weibull distribution is more plausible than the exponential. The $95 \%$ credible interval for the regression parameters describing treatment contrasts of interest did not include zero indicating toxicity of the chemical at all dose levels. This same final conclusion was reached by all models. However, the credible intervals were affected by the choice of the model.

As explained in Section 3, conventional analyses transform the tail intensities using logarithmic transformations. The mean of the transformed responses is then used as a summary measure for each rat. The hierarchical nature of the data is thus completely ignored and a simple analysis of variance is used to test whether there is a dose effect. Comparing this conventional model (Table 1) to our preferred model would be rather difficult since as we are using different responses and different type of models. We can, however, compare this with an equivalent model from our set of proposed models which completely ignores the hierarchical structure, but which uses the appropriate distribution and all the available information, namely the classical Weibull model. Upon comparison of the classical Weibull model and Model 8, the parameters of interest are highly significant in both cases. Yet, the standard errors, likewise the credible intervals of Model 8 are twice that of the classical Weibull model. While not the case in this example because of the high toxicity of the compound of interest, 
Table 4: Parameter estimates obtained from Weibull-Gamma(oD)-Gamma(RE1)-Gamma(RE2) and Weibull-Gamma(OD)-Normal(RE1)-Gamma(RE2) for Tail Length.

\begin{tabular}{lccccc}
\hline \hline & \multicolumn{2}{c}{ Weibull-G G G } & \multicolumn{2}{c}{ Weibull G N(RE1) G(RE2) } \\
\hline Effect & Parameter & Est.(s.e.) & $95 \%$ C.I. & Est.(s.e.) & $95 \%$ C.I. \\
\hline Vehicle & $\beta_{0}$ & $-30.44(0.6646)$ & {$[-31.74,-29.12]$} & $-30.54(0.8001)$ & {$[-32.01,-28.97]$} \\
Low versus vehicle & $\beta_{1}$ & $-11.99(0.4977)$ & {$[-12.95,-11.01]$} & $-12.02(0.5171)$ & {$[-13.05,-11.06]$} \\
Medium versus vehicle & $\beta_{2}$ & $-12.14(0.5061)$ & {$[-13.1,-11.12]$} & $-12.19(0.5298)$ & {$[-13.27,-11.23]$} \\
High versus vehicle & $\beta_{3}$ & $-12.57(0.4946)$ & {$[-13.54,-11.58]$} & $-12.63(0.5357)$ & {$[-13.75,-11.64]$} \\
Pos. control versus vehicle & $\beta_{4}$ & $-9.75(0.5523)$ & {$[-10.84,-8.65]$} & $-9.752(0.5617)$ & {$[-10.88,-8.68]$} \\
Weibull shape & $\rho$ & $10.71(0.2192)$ & {$[10.26,11.13]$} & $10.71(0.2727)$ & {$[10.17,11.22]$} \\
Precision of RE1 & $\frac{1}{d}$ & & & $32.14(168.1)$ & {$[1.27,323.2]$} \\
OD parameter & $\alpha_{1}$ & $0.894(0.0431)$ & {$[0.815,0.984]$} & $0.8942(0.0494)$ & {$[0.806,0.999]$} \\
RE1 parameter & $\alpha_{2}$ & $4.597(3.179)$ & {$[1.53,12.67]$} & & \\
RE2 parameter & $\alpha_{3}$ & $1.611(0.2985)$ & {$[1.10,2.25]$} & $1.578(0.3135)$ & {$[1.05,2.28]$} \\
\hline \hline
\end{tabular}

this suggests that ignoring the hierarchical structure and overdispersion could have major influence on the final conclusion. Significant estimates in the classical Weibull model may be insignificant in Model 8. In other words, a compound might be erroneously declared toxic.

Based on the analysis for tail intensity, more elaborate models did not outperform (not much improvement in terms of DIC). However, this was not the case for the second response, tail length. Based on the DIC, the most complicated model has the best fit, showing the importance of the hierarchical structure as well as overdispersion. Models with one hierarchical random effect were better fitting as compared to the classical Weibull model. Models with two random effect improved the fit further, and models with the complete hierarchical structure and overdispersion random effect appear to be best. Further, notice that the model with only an overdispersion random effect is better fitting than models with only the hierarchical structure, showing the importance of the overdispersion relative to the hierarchical structure.

Note the effects of the model on the parameter estimates. When only one hierarchical structure (one random effect) is added to the classical Weibull model, the point estimates were slightly higher and the standard error for the contrast of interest was approximately four times smaller. Smaller DIC for 
Table 5: Parameter estimates obtained from Weibull-Gamma(OD) and Weibull-Normal(RE1)Gamma(RE2) and Weibull for Tail Length.

\begin{tabular}{lcccc}
\hline \hline & & Weibull G(OD) & Weibull N(RE1) G(RE2) & Weibull \\
\hline Effect & Parameter & Est.(s.e.) & Est.(s.e.) & Est.(s.e.) \\
\hline Vehicle & $\beta_{0}$ & $-27.36(0.5968)$ & $-15.26(0.2519)$ & $-12.76(0.1543)$ \\
Low versus vehicle & $\beta_{1}$ & $-10.58(0.2612)$ & $-4.79(0.2468)$ & $-3.55(0.0530)$ \\
Medium versus vehicle & $\beta_{2}$ & $-10.76(0.2638)$ & $-4.89(0.2479)$ & $-3.65(0.0535)$ \\
High versus vehicle & $\beta_{3}$ & $-11.13(0.2705)$ & $-5.10(0.2509)$ & $-3.85(0.0550)$ \\
Pos. control versus vehicle & $\beta_{4}$ & $-8.55(0.2248)$ & $-3.79(0.3028)$ & $-2.70(0.0590)$ \\
Weibull shape & $\rho$ & $9.48(0.2152)$ & $4.96(0.0572)$ & $4.01(0.0422)$ \\
Precision of RE1 & $\frac{1}{d}$ & & $45.83(54.60)$ & \\
OD parameter & $\alpha_{1}$ & $0.8569(0.0435)$ & & \\
RE2 parameter & $\alpha_{3}$ & & $3.031(0.5393)$ & \\
\hline \hline
\end{tabular}

models with the second random effect (slide) showed the importance of slide effect in contrast to rat. Extending to two random effects, the standard error slightly increased further. The inclusion of an overdispersion random effect had a very important impact on the estimate (approximately 3 times) and standard error (four times) in contrast with the classical Weibull model. With the inclusion of one hierarchical random effect to the overdispersion, the standard error was doubled. Models with complete hierarchical structure and overdispersion yielded a slightly different estimate compared to the estimate from a model with overdispersion alone and a changing estimate (approximately 2.5 times) in contrast to the estimate of the corresponding models with two hierarchical random effects but no overdispersion; the standard error was double in contrast to both models. Generally, for tail length, we did not reach a different conclusion, due to high toxicity of the compound; however, inclusion of the hierarchical structure and overdispersion random effect had severe impact on the magnitude, standard errors as well as the credible intervals. Results for the classical Weibull model with overdispersion alone and a model with two hierarchical random effects are given in Table 5. 


\section{Concluding Remarks}

In this paper, we proposed a flexible modeling framework for the comet assay data using a Bayesian hierarchical model. It takes not only the complete hierarchical nature but also the appropriate non-Gaussian probability distribution for the response into account. It further includes a possible overdispersion that may exist in the data. Both normal and gamma random effects can be considered to account for clustering in the same framework, the more conventional models with either the overdispersion, or just one hierarchical random effect being submodels.

The method was applied to the comet assay data gathered to assess the toxicity of 1,2-Dimethylhydrazine dihydrochloride at different dose levels. For this particular dataset, a Weibull-gamma(RE2) model seemed adequate for tail intensity, whereas a Weibull-gamma(OD)-gamma(RE1)-gamma(RE2) was better fit for tail length. A comparison of these analysis with the conventional approach, which ignores the overdispersion and the hierarchy in the data, revealed that both models led to the same qualitative conclusion of severe toxicity of the compound at all dose levels. This notwithstanding, estimates, standard errors, and credibility intervals were severely affected, underscoring the risk of using models that are too simple. In general, proper models encompassing at the same time the hierarchical nature in the data, combined with overdispersion effects, need to be adopted. In this case, the use of the overdispersion and hierarchical structure improved the fit for one response. Furthermore, even when the more elaborate model does not provide a substantially improved fit, nor alters the inferences drawn, the development is still very useful because it provides further confidence, by way of model specification assessment, on the quality of the purported model.

\section{Acknowledgments}

Financial support from the IAP research network \#P6/03 of the Belgian Government (Belgian Science Policy) and from Fund for Scientific Research-Flanders (F.W.O.) is gratefully acknowledged.

\section{References}

Cox, D.R. and Hinkley, D.V. (1974) Theoretical Statistics. London: Chapman \& Hall/CRC. 
Duchateau, L. and Janssen, P. (2007) The Frailty Model. New York: Springer.

Ejchart, A. and Sadlej-Sosnowska, N. (2003). Statistical evaluation and comparison of comet assay results. Mutation Research, 534, 85-92.

Gelman, A. and Hill, J. (2006) Data Analysis using Regression and Multilevel/Hierarchical Models. New York: Cambridge University.

Kenward, M.G. and Roger, J.H. (1997) Small sample inference for fixed effects from restricted maximum likelihood. Biometrics, 53, 983-997.

Lee, Y., Nelder, J.A., and Pawitan, Y. (2006) Generalized Linear Models with Random Effects: Unified Analysis via H-likelihood. Boca Raton: Chapman \& Hall/CRC.

Lovell, D. P. and Omori, T. (2008) Statistical issues in the use of the comet assay.Mutagenesis, 23, $171-182$.

McCullagh, P. and Nelder, J.A. (1989) Generalized Linear Models. London: Chapman \& Hall.

Molenberghs, G. and Verbeke, G. (2005) Models for Discrete Longitudinal Data. New York: Springer.

Molenberghs, G., Verbeke, G., and Demétrio, C. (2007) An extended random-effects approach to modeling repeated, overdispersed count data. Lifetime Data Analysis, 13, 513-531.

Molenberghs, G., Verbeke, G., Demétrio, C.G.B., and Vieira, A. (2010) A family of generalized linear models for repeated measures with normal and conjugate random effects. Statistical Science, 00, 000-000. 


\section{A R2winbugs Code}

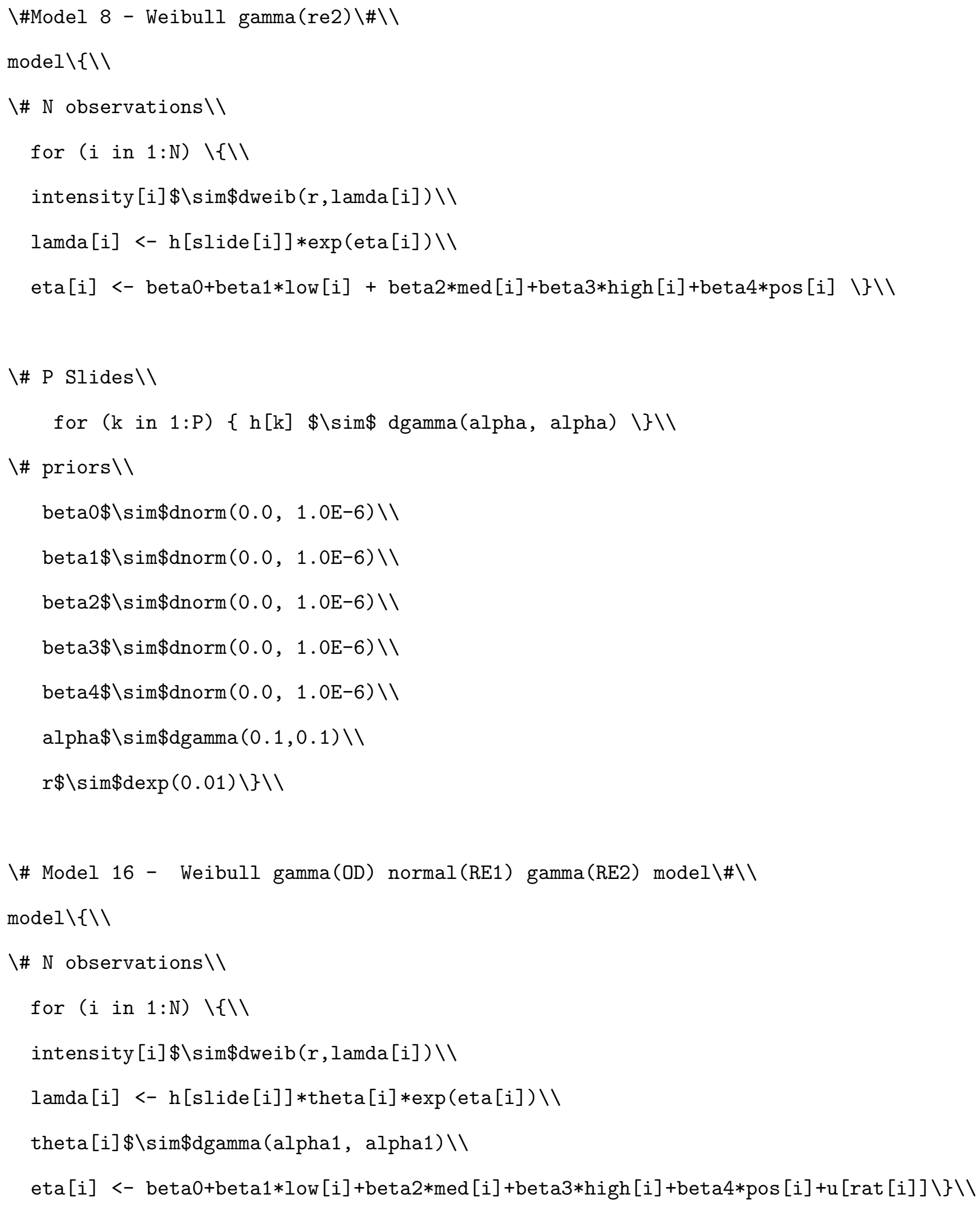




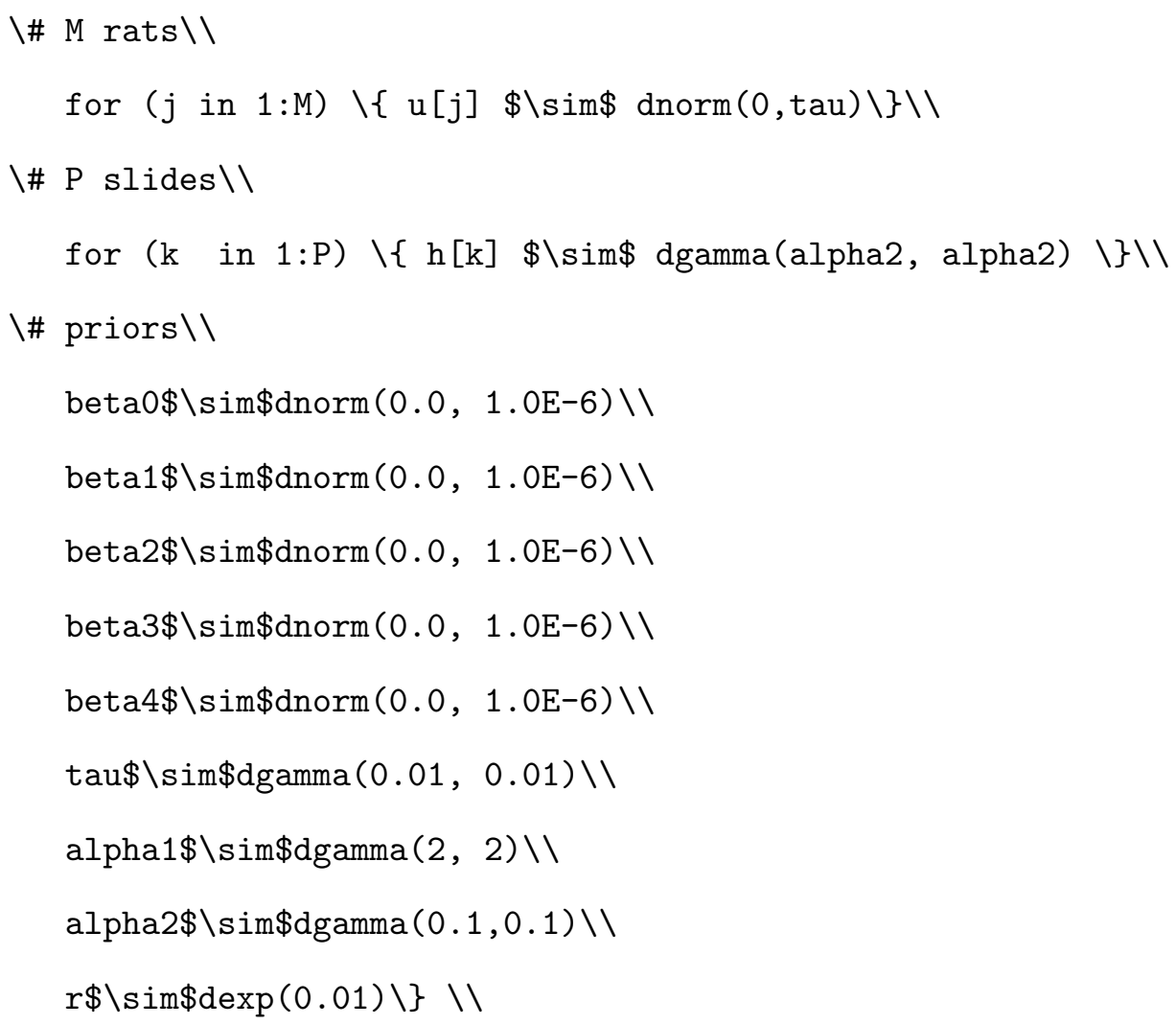

